\title{
Unambiguous Evidence of Two Plasmon Decay During Ultrafast Laser Writing in Glass
}

\author{
Aabid Patel, Mindaugas Gecevičius, Rokas Drevinskas, Martynas Beresna and Peter G. Kazansky \\ Optoelectronics Research Centre, University of Southampton, SO17 1BJ, United Kingdom \\ ap7g12@orc.soton.ac.uk
}

The interaction of femtosecond laser pulses with transparent media has been a focus of research due to its unique properties. It has been established that above a certain threshold, self-assembled nanogratings in silica glass can be induced [1]. Although the mechanism that triggers the nanostructure is still unclear, a theory has been introduced involving the mechanism of nanogratings formation based on two plasmon parametric decay [2]. A signature of two-plasmon decay is the generation of the $3 / 2$ harmonic. Previously, in transparent media, only a weak $3 \omega / 2$ emission was observed at high energy thresholds [3]. Thus it remains unclear if two plasmon decay can be associated with self-assembled nanogratings formation. Here we present a thorough survey of $2^{\text {nd }}$, $3^{\text {rd }}$ and $3 / 2$ harmonic generations in fused silica for varying laser fluences within multiple regimes of optical laser writing and self-assembled nanostructures. We demonstrate that $3 \omega / 2$ can be observed at the energies close to the threshold of permanent material modification.

Regeneratively amplified, mode-locked Yb:KGW based femtosecond laser system (Pharos, Light Conversion Ltd.) operating at $1030 \mathrm{~nm}$ and delivering pulses of $\sim 300 \mathrm{fs}$ at varying repetition rates was used in the experiments. The laser beam was focused into the bulk of the silica via a $\times 10(\mathrm{NA}=0.16)$ aspheric lens. A series of spectral measurements for varying pulse energy were taken translating the sample and at stationary positions and analysed with an Andor Shamrock SR-303i imaging spectrometer, where two gratings were used to vary the spectral range of the measurement (150 and 1200 lines/mm).

(a)

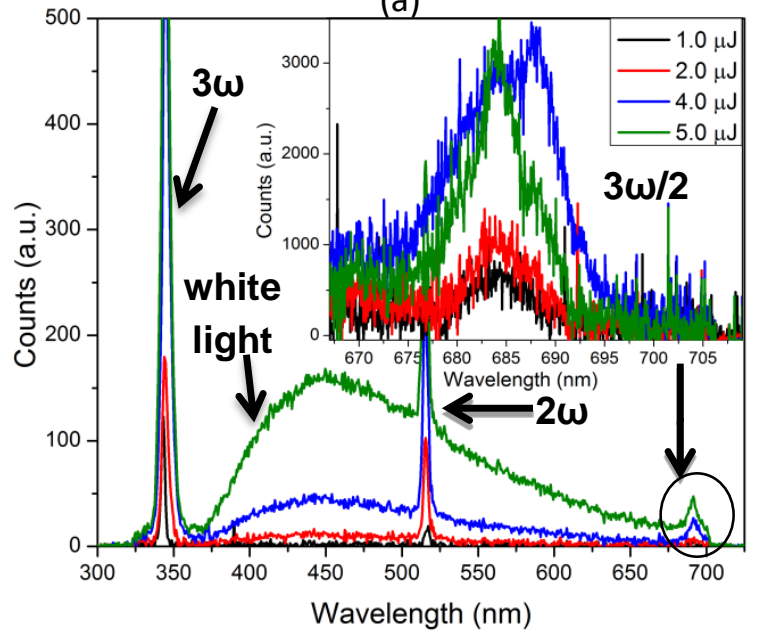

(b)

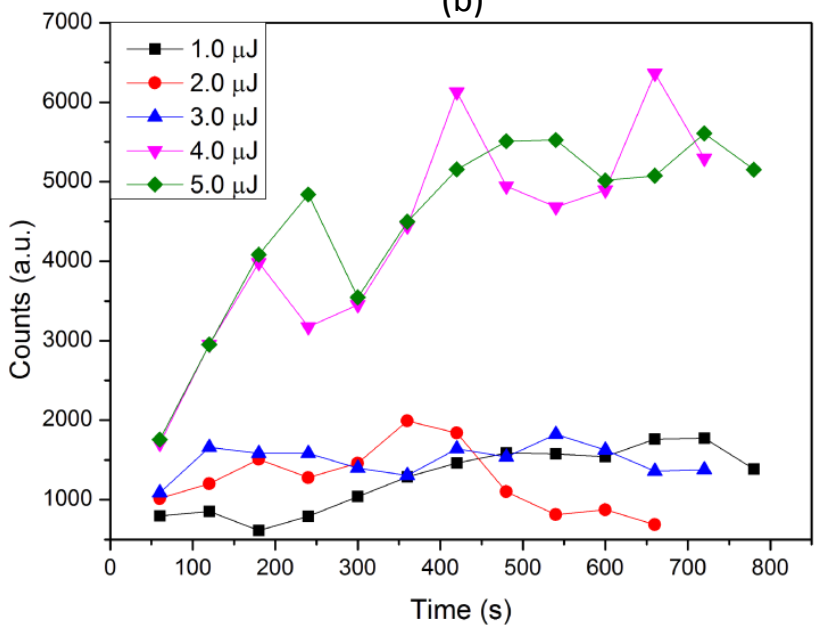

Fig. 1 a) Spectrum of harmonics for varying pulse energies (200 kHz repetition rate). White light emission and the double peak structure for $3 \omega / 2$ are observed as pulse energy is increased. b) Time dependence of $3 \omega / 2$ for varying pulse energies.

Analysing the light collected after the sample, we observed three distinctive peaks, corresponding to second, and third harmonics and $3 \omega / 2$. The spectrum measured for varying pulse energies revealed that $3 \omega / 2$ could be observed just above the permanent modification threshold, with a characteristic two-peak structure associated with forward and backward propagating plasmons and a bandwidth of $\sim 20 \mathrm{~nm}$ (Fig. 1a). All three harmonics were increasing with pulse energy and evolved on the time scale of several minutes (Fig. 1b). The amplitudes seen for $3 \omega / 2$ are constant for low pulse energies where self-assembled nanogratings are formed indicating that two plasmon decay can play an important role in the formation of nanostructures. At higher pulse energies where strong damage occurs, the signal becomes erratic. Additionally, the observed emission of the three halves harmonic clarifies a long standing issue on electron concentration values in ultrafast laser material modification regimes. Since the two plasmon decay is possible for the electron concentration $n_{e}=n_{c r} / 4[2,3]$, where $n_{c r}$ is the critical plasma density, we could state electron concentration is at least $4 \times 10^{20} \mathrm{~cm}^{-3}$.

\section{References}

[1] Y. Shimotsuma, P. G. Kazansky, J. Qiu, and K. Hirao, "Self-organized nanogratings in glass irradiated by ultrashort light pulses," Phys. Rev. Lett. 91, 247405 (2003)

[2] P. G. Kazansky, E. Bricchi, Y. Shimotsuma, and K. Hirao, "Self-Assembled Nanostructures and Two-Plasmon Decay in Femtosecond Processing of Transparent Materials," in Conference on Lasers and Electro-Optics/Quantum Electronics, (OSA, 2007), paper CThJ3. [3] M. Watanabe, S. Juodkazis, J. Nishii, S. Matsuo, and H. Misawa, "Microfabrications by a High Fluence Femtosecond Exposure: Mechanism and Applications," SPIE. 4637, 159 (2002). 